\title{
Factores Asociados al Burnout Académico en Estudiantes de Internado Profesional de Fonoaudiología
}

\section{ASSOCIATED FACTORS WITH ACADEMIC BURNOUT IN INTERN STUDENTS OF SPEECH LANGUAGE PATHOLOGY}

\author{
Virginia Andrea García-Flores', Yuri Esperanza Vega Rodríguez¹, Bárbara Libni Farías Fritz¹, Luis Rodolfo Améstica-Rivas², \\ Raquel Auristela Aburto Godoy ${ }^{3}$ \\ 1. Académica Departamento de Ciencias de la Rehabilitación en Salud; Facultad de Ciencias de la Salud y de los Alimentos; Universidad del Bío-Bío, Chillán, Chile. \\ 2. Académico Departamento de Gestión Empresarial, Facultad de Ciencias Empresariales; Universidad del Bío-Bío, Chillán, Chile. \\ 3. Unidad de Gestión Curricular y Monitoreo, Área de Desarrollo Pedagógico y Tecnológico; Universidad del Bío-Bío, Chillán, Chile.
}

\begin{abstract}
RESUMEN
Los estudiantes que cursan el internado profesional de fonoaudiología experimentan situaciones de estrés y agotamiento propias de esta actividad, pudiendo presentar burnout académico (BA), ya que el contacto con usuarios reales, la exigencia académica y las acciones propias de un contexto laboral son características de la práctica profesional. El objetivo del presente estudio es determinar la existencia del burnout académico y la asociación con variables sociodemográficas en los estudiantes de 5to año de fonoaudiología. Se aplicó un cuestionario a 47 sujetos de internado profesional para recopilar antecedentes personales, académicos y sociales. Luego, para evaluar el burnout académico, se aplicó el Maslach Burnout Inventory-Student Survey.

Los estudiantes evaluados no presentan el síndrome, aunque el factor cansancio emocional se presentó con más altos puntajes. Tampoco existen diferencias entre los puntajes del síndrome para los estudiantes que realizan su práctica en las áreas de Salud y Educación. Las variables explicativas del alto cansancio emocional en este estudio son el sexo masculino, pertenecer a sector urbano y aumento de consumo de tabaco. El estudio del BA es importante para tomar medidas preventivas en el transcurso de la vida universitaria para garantizar a futuro el compromiso y mejor desempeño por parte de los estudiantes que realizan su práctica profesional.
\end{abstract}

(García V, Vega Y, Farías B, Améstica L, Aburto R, 2018. Factores Asociados al Burnout Académico en Estudiantes de Internado Profesional de Fonoaudiología. Cienc Trab. May-Ago; 20 [62]: 84-89).

Palabras clave: BURNOUT ACADÉMICO, PRÁCTICA PROFESIONAL, FONOAUDIOLOGÍA.

\section{ABSTRACT}

Students who attend internship of speech language pathology experience situations of stress and exhaustion characteristic of this activity, being able to present academic burnout, due to the contact with real users, the academic requirement and the own actions of a work context, which are characteristics of the internship. The objective of this study was to determine the existence of academic burnout and its association with sociodemographic variables in the 5th year students of speech language pathology. A questionnaire was applied to 47 intern students in order to gather personal, academic and social backgrounds. After to evaluate the academic burnout, the Maslach Burnout Inventory - Student Survey was applied.

The students evaluated did not present the syndrome, although the emotional fatigue factor was presented with higher scores. There are also no differences in the scores of the syndrome for students who did their practice in both health and education setting. The explanatory variables of high emotional fatigue in this study were male sex, belonging to the urban sector and increased tobacco consumption. The BA study is important to take preventive measures over the university life to ensure future commitment and better performance by students who perform their professional practice.

Key words: ACADEMIC BURNOUT, INTERNSHIP, SPEECH LANGUAGE PATHOLOGY.

\section{INTRODUCCIÓN}

\section{Burnout y Burnout Académico}

En el ámbito laboral existen factores que intervienen en el desempeño de las personas. Estos factores son condiciones que se relacionan con la manera en la que está organizado el trabajo, el tipo

Correspondencia / Correspondence:

Flga. Mg. Virginia A. Garcia Flores.

Av. Andrés Bello $N^{\circ} 720$, Campus Fernando May; Chillán, Chile e-mail:vgarcia@ubiobio.cl

Tel.: 422463251

Recibido: 11 de Enero 2018 / Aceptado: 29 de Abril 2018 de puesto, la realización de la tarea, e incluso con el entorno. ${ }^{1}$ Los factores psicosociales pueden tener diferentes impactos en la calidad de vida laboral de las personas, pudiendo llegar a favorecer o a perjudicar las actividades laborales. En este ámbito aparece el termino Burnout acuñado en 1974 por Freudenberger, y se define como un síndrome identificado dentro de la organización laboral, el cual por tener una naturaleza psicosocial, afecta el funcionamiento en el trabajo y la salud de las personas. Este refleja el desgaste experimentado por los profesionales de los servicios humanos, quienes se desempeñan en atención directa con personas como lo es en ámbitos de acción de las ciencias de la salud, educación y ciencias sociales. ${ }^{2}$ En este sentido, los estudiantes de carreras de la salud y de la educación al enfrentarse en los últimos años de su carrera al internado o prácticas profesionales, pueden llegar a experimentar sensaciones y percepciones de no poder dar 
más de sí mismos, tanto física como psíquicamente, asumiendo actitudes negativas, pérdida de interés o del valor de los estudios, desarrollando así dudas sobre la propia capacidad de realizarlos. ${ }^{3}$ A todas estas características es a lo que se le llama Burnout Académico (BA). El Síndrome de Burnout (SB) es caracterizado como un estrés crónico evidenciado en: agotamiento o cansancio emocional, pérdida de interés y atractivo por la actividad que se desempeña; en despersonalización, evidenciada en actitudes impersonales y negativas hacia los usuarios; y sentimiento de inadecuación a la actividad ocupacional, evidenciada en un sentimiento y creencia de que el trabajo no vale la pena. ${ }^{4}$

Son varios los estudios que han observado el BA en estudiantes universitarios..$^{3,5-10}$ Este ámbito es considerado como uno de los más vulnerables para que el burnout aparezca a partir de diferentes factores tanto de la organización como personales de interacción, puesto que los estudiantes se ven sometidos al igual que los profesionales a sobrecargas y exigencias propias del contexto en el que se desenvuelven. ${ }^{4}$

Si bien en el último año de la carrera los internos aún son estudiantes, el contexto demanda un gran compromiso laboral, el cual es equiparable con el de un profesional, encontrándose expuestos a situaciones de estrés laboral y al cansancio y desgaste derivado de este. Estas demandas inciden en el funcionamiento académico del estudiante determinándose por una escasa productividad académica, déficit de atención, concentración, memorización, en la resolución de problemas y toma de decisiones efectivas. ${ }^{3}$ De la misma forma se han encontrado resultados de burnout en estudiantes, relacionados con factores como sintomas de depresión, consumo de bebidas alcohólicas, drogas, estimulantes, nicotina ${ }^{11} \mathrm{y}$ ansiedad. $^{3}$

Burnout Académico y variables académicas, personales y sociales El BA, al ser un estado mental persistente, negativo, caracterizado por el agotamiento emocional, distress en las actividades académicas, hace que los estudiantes evidencien poca motivación frente a los estudios. ${ }^{2}$ Los estudiantes perciben que son incapaces de superar los retos y enfrentar las exigencias en el quehacer académico, lo que conlleva a una baja autoeficacia. Esta autoeficacia no solo aparece como una variable predictora en la aparición de burnout, sino también relacionada con el rendimiento académico $^{2,12}$ afectando de manera importante el proceso de aprendizaje. Características del BA como el sentirse ineficaz, experimentar agotamiento y la presencia de cinismo son aspectos que influyen en el rendimiento académico por la falta de satisfacción. ${ }^{12}$ Coincidente con lo anterior en que los estudiantes responden a los compromisos y responsabilidades académicas presentando BA, se observa una baja percepción de eficacia, un bajo rendimiento académico, pérdida de semestres y asignaturas conllevando una tendencia a la deserción. ${ }^{2}$

En otros estudios se señalan variables del individuo que pueden predecir o ser factores de riesgo en la población académica, como son el sexo -las mujeres se perciben más eficaces y los hombres puntúan más alto en cinismo-; las características de personalidad como ansiedad de rasgo, rigidez, perfeccionismo y baja autoeficacia son más vulnerables de sufrir burnout. ${ }^{5}$ Estudios evidencian que las mujeres suelen ser menos autoeficaces que los hombres. ${ }^{2}$ Un estudio de Reyes y Medina ${ }^{11}$ reportó que ciertas variables aparecen como factores protectores y otros como factores de riesgo: las mujeres tuvieron 2.2 veces más riesgo de padecer el SB. Por otro lado, variables como el abuso de sustancias también han sido estudiadas, donde se reporta mayor presencia de BA en los que vivian solos, no realizaban actividad extracurricular, consumían más de una vez a la semana alcohol, no recibían remuneración, trabajaban más de 8 horas diarias, presentaban alguna enfermedad mental y tenían relaciones malas-regulares con el equipo de trabajo. ${ }^{13}$ Otra investigación reveló relación del SB con algunos estilos de comportamiento: mayor severidad del SB aumentaba el consumo de alcohol, nicotina, estimulantes y cafeína; esta última con significancia estadística $(\mathrm{p}=0004) .{ }^{11}$

En otros estudios se encontró relación entre la institución de procedencia, estado civil, escolaridad y antigüedad en la institución con el componente agotamiento emocional del síndrome. Solo la escolaridad (superior a la requerida para el puesto) y el estado civil (ser soltero) fueron factores de riesgo para el síndrome, específicamente con la dimensión agotamiento emocional. ${ }^{14}$

En resumen, la presencia del BA se puede relacionar con distintas variables personales, sociales y académicas que pudieran actuar como factores de riesgo o protectores en los individuos; por lo tanto, es importante considerar estas y otras variables en estudios respecto al BA para poder tomar medidas de precaución y control en el proceso de enseñanza aprendizaje de los estudiantes universitarios.

\section{MATERIAL Y MÉTODOS}

\section{Participantes}

El trabajo se enfocó en los estudiantes de quinto año de la carrera de fonoaudiología de una universidad estatal chilena que cursaron su práctica clínica durante el año 2016, encuestando a los 50 estudiantes matriculados, eliminándose a aquellos que presentaban más de un 10\% de respuestas perdidas en los instrumentos, obteniéndose una muestra válida de 47 sujetos, lo cual representa el 94\%.

\section{Instrumentos}

Se aplicó un cuestionario sociodemográfico diseñado para recopilar antecedentes personales (p. e.: sexo, edad, estado civil), características académicas (p. e.: promedio de notas, horas de trabajo autónomo, procedencia colegio) y características sociales (p. e.: consumo de tabaco, alcohol, origen residencia).

Para evaluar el BA, se aplicó el Maslach Burnout InventoryStudent Survey. Los estudiantes debieron responder a 22 afirmaciones en relación a los sentimientos que experimentan con su práctica profesional, la cual realizan en dos áreas: Salud y Educación. Las posibilidades son: nunca (0), pocas veces al año o menos (1), una vez al mes o menos (2), unas pocas veces al mes (3), una vez a la semana (4), pocas veces a la semana (5), todos los días (6). En las tres escalas la puntuación mínima es de 0 , mientras que la máxima es de 54 para cansancio emocional, 30 para despersonalización y 48 para realización personal, clasificándolos en tres niveles: bajo, medio y alto de acuerdo a los puntos de corte de Seisdedos y que se detallan en Tabla 1.

\section{Tabla 1.}

Escala puntos de corte síndrome de burnout.

\begin{tabular}{llcc} 
Dimensión & \multicolumn{1}{c}{ Alto } & Medio & Bajo \\
Cansancio emocional & mayor o igual a 27 & entre 19 y 26 & menor a 19 \\
Despersonalización & mayor o igual a 10 & entre 6 y 9 & menor a 6 \\
Baja realización personal & menor a 34 & entre 34 y 39 & mayor o igual a 40
\end{tabular}

Fuente: elaboración propia a partir de Seisdedos (1997). 


\section{Técnicas de análisis de los datos}

Para analizar las respuestas de los participantes en la encuesta, en una primera parte se aplicó estadística descriptiva, usando el programa estadístico SPSS 18. En una segunda parte, se realizó un análisis factorial.

Para determinar la fiabilidad del instrumento en su totalidad (44 elementos), como también de cada una de las dimensiones (D) que agrupó un conjunto de preguntas de percepción, se determinó el indicador Alpha de Cronbach. Para la totalidad del instrumento de diagnóstico el indicador fue altamente aceptable, con un resultado de 0,921 (ver Tabla 2, Panel A), bajo los criterios de George \& Mallery. ${ }^{15}$ Para las dos dimensiones estudiadas, los resultados fueron superiores a 0,80, siendo aceptable (ver Tabla 2, Panel B).

Tabla 2.

Estadísticos de fiabilidad del instrumento.

\begin{tabular}{|c|c|c|}
\hline \multicolumn{3}{|c|}{ Panel A: Estadístico de fiabilidad para el instrumento. } \\
\hline Dimensión & Alfa de Cronbach & $\mathrm{N}^{\circ}$ de Elementos \\
\hline Totalidad del instrumento & 0,921 & 44 \\
\hline \multicolumn{3}{|c|}{ Panel B: Estadísticos de fiabilidad para las dos dimensiones (D) del instrumento } \\
\hline Dimensión & Alfa de Cronbach & $\mathrm{N}^{\circ}$ de Elementos \\
\hline D1: Percepción MBI - Salud & 0,822 & 22 \\
\hline D2: Percepción MBI - Educación & 0,879 & 22 \\
\hline
\end{tabular}

Fuente: elaboración propia

Asimismo, al realizar las pruebas de KMO y Barlett para evaluar la significancia estadística del instrumento aplicado, se obtuvo un KMO para cada dimensión, lo que indica una alta relación entre las variables (Tabla 3). Por otra parte, al observar la prueba de esfericidad de Barlett se apreció una significancia menor a 0,05, por lo cual el modelo es estadísticamente significativo.

Tabla 3.

Pruebas KMO y esfericidad de Barlett.

\begin{tabular}{llccc} 
Dimensión & KMO & $\begin{array}{c}\text { Aprox. } \\
\text { Chi-cuadrado }\end{array}$ & gl & Sig. \\
\hline D1: Percepción MBI - Salud & 0,677 & 606,703 & 231 & 0,000 \\
D2: Percepción MBI - Educación & 0,641 & 708,028 & 231 & 0,000
\end{tabular}

Fuente: elaboración propia

\section{RESULTADOS}

De los 47 participantes, un 76,6\% son mujeres y el 23,4\% hombres, con una edad promedio de 24 años. En cuanto el estado civil, el 68,1\% reportó ser soltero/a sin hijos; en pareja sin hijos, $21,3 \%$ y otro no informó su estado civil, con 10,6\%. El 74,5\% proviene del sector urbano y 25,5\% del sector rural. Además, el $72,3 \%$ aporta a su ingreso económico familiar y el 80,8\% declara tener uno o dos hermanos. En cuanto al tipo de familia, un 29,8\% declara monoparental, un 66,0\% nuclear y un 4,3\% dice pertenecer a familia extensa.

Del total, un 63,3\% provenía de establecimientos particulares subvencionados, un 29,8\% de establecimientos municipalizados y de 6,4\% particular pagados (privados). El 89,4\% declara que es su primera carrera en la universidad. El 55,3\% declara realizar actividades extraprogramáticas en su acividad académica. En la universidad, el promedio de horas de trabajo autónomo del grupo es de un 7,45 horas, con un mínimo de 1 y máximo de 45 , en tanto, el 61,7\% declara menos de 5 horas de trabajo autónomo.
La mayoría de los participantes declara que en su tiempo libre se dedica generalmente a trabajar, descansar, estar con la familia y leer. El 91,5\% declara contar con una red de amigos, el 17,7\% declara tener problemas de salud permanente contra un 83,3\% que dice no tenerlo. El 53,2\% declara no consumir bebidas alcohólicas, un 29,8\% declara que sólo los fines de semana, en tanto, el 78,7\% declara no fumar y, una vez a la semana, un 12,8\%.

Los estudiantes declaran que el ámbito que más tiempo les demanda es Educación con 55,3\% frente a Salud con un 44,7\%. En virtud de su trabajo de clínica, la "rotación” que más les estresó fue la primera, con un 55\%, seguida por la segunda rotación con un $23,4 \%$, la cuarta con un $17 \%$ y la tercera con un $8,5 \%$. Una vez pasado por los internados, el 68,1\% declara que el área salud es más estresante. El 89,4\% de los participantes declara haberse sentido estresado.

En cuanto a los resultaddos obtenidos en la aplicación del instrumento Maslach Burnout Inventory-Student Survey, se realizó una evaluación de la consistencia interna de las puntuaciones de los instrumentos aplicados mediante el coeficiente alfa de Cronbach y un análisis descriptivo de los puntajes de cada factor usando media, desviación estándar, mínimo y máximo. En las tablas 4 y 5 se observan los niveles de burnout de los estudiantes en las áreas Salud y Educación. La confiabilidad en el área Salud presenta una consistencia interna suficiente para su uso en investigación en los tres factores, aunque se muestra levemente inferior en el factor realización personal. En el caso del área Educación, el factor despersonalización muestra una menor confiabilidad, pero aceptable.

En la misma línea investigativa de Glaria et $\mathrm{al}^{6}$, calcularon el coeficiente $\mathrm{M} / \mathrm{i}$ (media aritmética dividida por el número de ítems del factor) a fin de poder realizar una comparación heurística entre estos, encontrándose que los estudiantes reportan un mayor nivel de cansancio emocional $(\mathrm{M} / \mathrm{i}=3.03)$ que de las otras manifestaciones del burnout.

Tabla 4.

Maslach Burnout Inventory aplicado en área Salud.

$\begin{array}{lcccccc}\text { Dimensiones } & \text { Alfa } & \text { Media } & \text { D. E. } & \text { Mín. } & \text { Máx. } & \text { M/i } \\ \text { Cansancio emocional } & 0,894 & 27,2766 & 12,44589 & 5,00 & 51,00 & 3,03 \\ \text { Despersonalización } & 0,807 & 6,5532 & 6,93388 & 0,00 & 24,00 & 1,31 \\ \text { Realización personal } & 0,754 & 37,9787 & 6,46223 & 22,00 & 48,00 & 4,75\end{array}$

Fuente: elaboración propia.

Tabla 5.

Maslach Burnout Inventory aplicado en área Educación.

$\begin{array}{lcccccc} & \text { Alfa } & \text { Media } & \text { D. E. } & \text { Mín. } & \text { Máx. } & \text { M/i } \\ \text { Cansancio emocional } & 0,913 & 26,0213 & 13,14880 & 0,00 & 54,00 & 2,89 \\ \text { Despersonalización } & 0,769 & 6,2766 & 6,60600 & 0,00 & 25,00 & 1,26 \\ \text { Realización personal } & 0,872 & 36,4255 & 8,63951 & 6,00 & 48,00 & 4,55\end{array}$

Fuente: elaboración propia.

Posteriormente, con el fin de comparar las dos muestras relacionadas, Salud y Educación, se realizó una prueba de medias la cual nos permite contrastar hipótesis sobre igualdad de medias, según Tabla 6. En primer lugar, se comparó el puntaje obtenido en el factor cansancio emocional entre ambas áreas, no encontrando diferencias estadísticamente significativas (Sig. ,218), con un coeficiente de correlación 0,857 (Sig. 000); lo mismo ocurrió en despersonalización, donde no se encontró diferencias significativas (Sig. ,623), con una correlación de 0,841 (Sig. 000) y lo 
Tabla 6.

Prueba de muestras entre Salud y Educación.

$\begin{array}{lccc}\text { Dimensión y área } & \mathrm{t} & \mathrm{gl} & \text { Sig. (bil.) } \\ \text { Cansancio emocional Salud-Educación } & 1,250 & 46 & 0,218 \\ \text { Despersonalización Salud-Educación } & 0,496 & 46 & 0,623 \\ \text { Baja realización personal Salud-Educación } & 1,883 & 46 & 0,066\end{array}$

Fuente: elaboración propia.

Tabla 7.

Número de estudiantes según escala de burnout.

\begin{tabular}{lccccccccc}
\multirow{2}{*}{ Dimensión } & \multicolumn{4}{c}{ Área Salud } & \multicolumn{4}{c}{ Área Educación } \\
& Alto & Medio & Bajo & N & Alto & Medio & Bajo & N \\
Cansancio emocional & 28 & 6 & 13 & 47 & 22 & 13 & 12 & 47 \\
Despersonalización & 14 & 6 & 27 & 47 & 11 & 9 & 27 & 47 \\
Baja realización personal & 14 & 12 & 21 & 47 & 15 & 10 & 22 & 47
\end{tabular}

Fuente: elaboración propia.

Tabla 8.

Variables explicativas alto cansancio emocional.

$\begin{array}{lcc}\text { Coeficientes } & \text { Modelo Área Salud } & \text { Modelo Área Educación } \\ \text { Caracteristicas personales } & & \\ \text { Intercepto } & -12,606(0,055) * * * & -4,015(0,454) \\ \text { Sexo } & 1,725(0,045)^{* * *} & 0,51(0,513) \\ \text { Edad } & 0,392(0,11) & -0,026(0,897) \\ \text { Estado Civil } & 0,238(0,507) & 0,205(0,552) \\ \text { Cantidad hermanos } & 0,549(0,206) & 1,025(0,025)^{* *} \\ \text { Tipo de familia } & 0,252(0,706) & 0,888(0,197) \\ \text { Características académicas } & & \\ \text { Intercepto } & 6,276(0,313) & 2,271(0,691) \\ \text { Promedio nota ex ante } & -0,717(0,564) & -0,223(0,847) \\ \text { Horas trabajo autónomo } & 0,064(0,235) & 0,09(0,832) \\ \text { Actividades extra- programáticas } & 0,928(0,198) & -0,538(0,396) \\ \text { Procedencia colegio } & -1,331(0,059)^{* * *} & -0,479(0,418) \\ \text { Caracteristicas sociales } & & \\ \text { Intercepto } & 3,202(0,101) & 2,412(0,173) \\ \text { Consumo tabacos } & 1,226(0,073)^{* * *} & 0,721(0,164) \\ \text { Consumo bebidas alcohólicas } & -0,796(0,092)^{* * *} & -0,515(0,245) \\ \text { Problemas de salud } & -1,505(0,151) & -1,142(0,199) \\ \text { Residencia durante internado } & -0,173(0,650) & -0,116(0,740) \\ \text { Origen residencia } & -1,565(0,049)^{* *} & -1,178(0,129) \\ \text { Estadistico t entre paréntesis } & & \\ \text { *significativa al nivel } 0,01 ;{ }^{* *} \text { significativa al nivel } 0,05 ; * * * \text { significativa al nivel } 0,10\end{array}$

mismo ocurrió en el factor en realización personal $(0,66)$, con una correlación de 0,756. En forma complementaria se realizó la Prueba de Kolmogorov-Smirnov, encontrando normalidad en la muestra.

En un segundo nivel de análisis y con el objetivo de visualizar en qué dimensión podría existir BA, se clasificaron los puntajes de los estudiantes de fonoaudiología (47) por cada una de las dimensiones separadas por área (Tabla 7). Se pudo determinar que la dimensión "Cansancio emocional" fue aquella donde los puntajes dan cuenta de una condición "Alto" en ambas áreas. Es así como en Salud, 28 estudiantes tuvieron un puntaje alto, lo que representa un 59,6\% del total. En tanto en el área de Educación, la condición de alto alcanzó a 22 estudiantes, lo que representa un $46,8 \%$ del total.

Al revisar la condición de bajo burnout, se da coincidentemente en ambas áreas en la dimensión "Despersonalización”, concentrando un 57,4\% del total de estudiantes.

A partir del encasillamiento realizado anteriormente, donde se estableció que la dimensión "Cansancio emocional" fue la que concentró la condición "Alto" en la mayoría de los estudiantes y ambas áreas, se procedió a realizar una regresión logística (binomial) a dicha dimensión, que permita conocer aquella variable o variables explicativas para ese nivel, definiendo con 1 la condición de alto y 0 la condición medio o bajo, coherente con al definición de Seisdedos. Los resultados de esta regresión logística se observan en Tabla 8, donde se estableció una clasificación por características personales, académicas y sociales, construyendo para cada una de las áreas los modelos con sus respectivas variables.

\section{DISCUSIÓN DE RESULTADOS}

La presente investigación se enfocó en el 5to año de la Carrera de Fonoaudiología, año en el cual el estudiante se enfrenta a su práctica profesional donde debe hacer "práctica la teoría" adquirida en los años anteriores, enfrentándose a usuarios reales y acciones propias de un contexto laboral, tanto en las áreas de salud y educación del Internado Profesional. El escenario actual de este grupo se ha caracterizado por un aumento de licencias médicas, mayor reprobación y alta deserción, evidenciando señales de agotamiento y estrés, características propias del BA.

Con el fin de determinar la existencia de burnout en los estudiantes practicantes de fonoaudiología, se adoptó los puntos de corte de Seisdedos desde una perspectiva dicotómica (sí o no), se pudo establecer que en el área de Salud, los estudiantes no presentan el sindrome, aunque el factor cansancio emocional presentó una media de 27,2766, puntaje que lo ubica levemente en el nivel alto que debe ser superior o igual a 27; despersonalización con una media de 6,5532 no siendo mayor o igual a 10 y baja realización personal, con una media de 37,9787 con un puntaje no menor a 34 puntos, pudiéndose inferir que en la totalidad se encuentra en una escala media de burnout. Lo que coincide con el estudio Ferrel16 en estudiantes de Ingeniería y Ciencias de la Salud, donde los resultados indican la no presencia del síndrome, pero existe una tendencia a presentarlo, evidenciándose altos porcentajes de cansancio emocional, mayoritariamente.

En el caso de la práctica en el área de Educación, los estudiantes en todos los factores se encuentran en un nivel medio no presentando el síndrome: el factor cansancio emocional presentó una media de 26,0213, bajo los 27 puntos de corte; despersonalización, con una media de 6,2766, con un puntaje bajo los 10 puntos de corte; y baja realización personal, con una media de 36,4255, puntaje superior a los 34 puntos de corte. En términos promedio, los estudiantes podrían ser encasillados en un nivel medio del síndrome.

Dada las pruebas de medias y no encontrando diferencias estadísticamente significativas en ninguno de los tres factores, se puede establecer que no existe diferencias en los puntajes del síndrome para los estudiantes que realizan su práctica en las áreas de Salud y Educación.

Debido a la no presencia del BA y siendo el cansancio emocional el que se presenta más alto dentro de los tres factores estudiados, se tomó este como elemento de análisis para las distintas variables. En relación a las variables explicativas del índice de cansancio emocional, se observa que las mujeres presentan menos cansancio emocional, lo cual se condice con lo encontrado por Breso ${ }^{5}$ respecto de variables predictoras en estudiantes universitarios: "las mujeres suelen percibirse más eficaces"; además, Ferrel ${ }^{16}$, en cuanto a la dimensión de autoeficacia, encontró una leve dife- 
rencia en las mujeres, siendo estas más autoeficaces, mostrando mejor desempeño y mejores expectativas de éxito. Otros estudios se condicen mostrando que las mujeres se percibían más autoeficaces en comparación con los hombres $;^{17,18}$ a diferencia de estos hallazgos, un estudio realizado en una muestra de estudiantes de odontología, enfermería, psicología de primero a séptimo año de medicina de una universidad chilena, mostró un nivel de prevalencia de estrés académico percibido del 36,3\%, mayor en mujeres que en hombres. ${ }^{8}$ A partir de los antecedentes no se observan diferencias concluyentes respecto a la variable sexo en cuanto a la presencia de BA.

Respecto de procedencia, en este estudio se presentan niveles de cansancio emocional más altos en los sujetos que provienen de colegios particulares que los de colegios municipales, así como los estudiantes cuya procedencia es urbana también evidencian un aumento en el cansancio emocional. La asociación entre el BA, colegio de procedencia y residencia originaria no se ha estudiado anteriormente, sólo se ha hecho referencia a la variable sociodemográfica de nivel socioeconómico. Asumiendo que los sujetos de origen rural y de colegios municipales pertenecen a niveles socioeconómicos más bajos, se condice con un estudio donde esos sujetos tendrian índices más bajos del BA. ${ }^{9}$ En dicho estudio el BA se asocia exclusivamente con padres que pagan los estudios de los estudiantes, y bajos índices de BA se asocia con estudiantes que pagaban ellos mismos sus estudios, lo que implicaría un mayor compromiso y esfuerzo propio.

En cuanto al consumo de sustancias, una investigación respecto de factores asociados con el SB en practicantes internos de hospital, encontraron síntomas de depresión, burnout, y consumo de licor en estudiantes de medicina; ${ }^{11}$ en otro estudio de Salud mental y predisposición de SB en estudiantes de enfermería, se señala que la tensión lleva al burnout, a la depresión y al abuso de sustancias. ${ }^{19}$ Por otro lado, la combinación de un alto nivel de agotamiento escolar y un bajo nivel de participación en el trabajo escolar, además del bajo rendimiento académico, son factores de riesgo importantes para fumar diariamente. ${ }^{20}$ En contraste con el presente estudio, al aumentar la cantidad de veces que fuma el estudiante por semana aumenta también el índice de cansancio emocional. Por el contrario, se observó una disminución en el cansancio emocional a medida que el estudiante consume alcohol más veces por semana. Haciendo un análisis de los datos pareciese ser que el consumo de alcohol, al estar supeditado a instancias sociales, pudiera ser un elemento desestresante dentro de las variables que conforman el síndrome.

Como lo menciona Bolaños ${ }^{21}$, otro factor que se relaciona con el BA es el desempeño académico, puesto que este se ve afectado por los bajos niveles de atención y concentración; en el presente estudio la variable "promedio notas" no presentó significancia estadística como explicativa del alto cansancio emocional.

El burnout en el ámbito académico en fonoaudiología ha sido poco estudiado, por lo que es importante conocer que factores están asociados e influyen positiva o negativamente en su manifestación. Si bien en el presente trabajo se estudió el BA, es el cansancio emocional el que presenta puntajes mayores de los tres factores estudiados. Es importante el análisis tanto de esta dimensión como de todo el síndrome de BA que permita generar apoyos preventivos y paliativos para un manejo adecuado de las manifestaciones del síndrome, facilitando así el proceso de formación académica de los estudiantes. Se deben encontrar técnicas que brinden herramientas a los estudiantes para generar habilidades que les ayuden a afrontar los altos niveles de estrés que surgen como resultado del proceso de enseñanza, de modo que no presenten el síndrome de burnout académico. ${ }^{21}$

Finalmente se hace necesario estudiar todos los niveles a lo largo de la carrera, ya que si bien es en quinto año donde tienen contacto con la atención de personas, es importante generar estrategias sistematizadas desde los primeros años de estudio, considerando en los estudiantes el desarrollo de un compromiso permanente con los usuarios que requerirán de sus servicios como profesionales, y de esta forma disminuir, por una parte, los indicadores no sólo de cansancio emocional sino las dimensiones del BA, y, por otro lado, las licencias psiquiátricas y deserción que aluden al estrés presentado en el último año de la carrera. 


\section{REFERÊNCIAS}

1. Gil-Monte PR. Riesgos Psicosociales en el Trabajo y Salud Ocupacional. Rev Peru Med Exp Salud Pública [en línea]. 2012 [citado jun 2018]; 29(2):237-241. Disponible en: http://www.ncbi.nlm.nih.gov/pubmed/22858772

2. Caballero CC, Hederich C, Palacio JE. El burnout académico: delimitación del sindrome y factores asociados con su aparición. Rev Latinoam Psicol. 2010; 42(1):131-146.

3. Palacio JE, Caballero CC, Gonzalez O, Gravini M, Contreras KP. Relación del burnout y las estrategias de afrontamiento con el promedio académico en estudiantes universitarios. Univ Psychol. 2012; 11(2):535-544.

4. Cabanach $R$, Gestal $A$. Regulación emocional y burnout académico en estudiantes universitarios de Fisioterapia. Rev Invest Educ [en línea]. 2011 [citado jun 2018]; 9(2):7-18. Disponible en: http://dialnet.unirioja.es/servlet/ articulo?codigo $=4730795$

5. Breso $E$, Caballero $C$, González 0 . Burnout en estudiantes universitarios. Psicol desde el Caribe. 2015; 32(3):424-441.

6. Glaría López R, Carmona San Martín L, Pérez Villalobos C, Parra Ponce P. Burnout y engagement académico en fonoaudiología. Investig Educ Med [en línea]. 2015 [citado jun 2018]; 5(17):17-23. Disponible en: http://www.sciencedirect.com/science/article/pii/S2007505715000447

7. Tomaschewski-Barlem JG, Lunardi VL, Ramos AM, da Silveira RS, Barlem ELD, Ernandes CM. Manifestações da sindrome de burnout entre estudantes de graduação em enfermagem. Texto Context-Enferm. 2013; 22(3):754-762.

8. Polanco A, Ortiz L, Pérez C, Parra P, Fasce E, Matus 0 , et al. Relación de antecedentes académicos y expectativas iniciales con el bienestar académico de alumnos de primer año de medicina. FEM [en línea]. 2014 [citado jun 2018]; 17(4):205-211. Disponible en: http://scielo.isciii.es/scielo.php?script=sci_ arttext\&pid=\$2014-98322014000400006\&lng=es\&nrm=iso\&tlng=es

9. Caballero CC, Hederich C, Garcia A. Relación entre BURNOUTy ENGAGEMENT académicos con variables sociodemográficas y académicas. Psicol desde el Caribe. 2015; 32(2):254-267.

10. Rodríguez G, Juan J, Manzanares L, Teresa M, Luque F. Los estudiantes de Grado en Enfermería y su compromiso con los estudios. Students in graduate programs in nursing and commitment to studies. Enferm Glob [en línea]. 2015 [citado may 2018]; 38:169-177. Disponible en: http://revistas.um.es/eglobal/article/ viewFile/198261/174161

11. Reyes Ticas A, Medina MT, Mesa X, Paredes Y, Barahona Y, Sierra M. Estudio de sindrome de "burnout", depresión y factores asociados en los practicantes internos del Hospital Escuela. Rev Fac Cienc Med (Tegucigalpa). 2012; $9(1): 14-20$.

12. Caballero CC, Abello R, Palacio J. Relación del burnout y el rendimiento académico con la satisfacción frente a los estudios en estudiantes universitarios. Av Psicol Latinoam. 2007; 25(2):98-111.

13. Estela-Villa LM, Jiménez-Román CR, Landeo-Gutiérrez JS, Tomateo-Torvisco JD, Vega-Dienstmaier JM. Prevalencia de síndrome de burnout en alumnos del séptimo año de medicina de una universidad privada de Lima, Perú. Rev Neuropsiquiatr [en línea]. 2010 [citado may 2018]; 73(4):147-56. Disponible en: http://www.upch.edu.pe/famed/revista/index.php/RNP/article/view/582

14. Picasso-pozo M, Huillca-Castillo N, Ávalos-Márquez J, Omori-Mltumori E, Gallardo-schultz A, Salas-chavez M. Sindrome de Burnout en Estudiantes de Odontologia de una Universidad Peruana. Kiru [en línea]. 2012 [citado may 2018]; 9(1):51-58. Available from: http://www.usmp.edu.pe/odonto/ servicio/2012/Kiruv.9/Kiru_v.9_Art8.pdf

15. George D, Mallery P. SPSS for Windows step by step: A simple guide and reference. 11.0 update. 4th ed. Boston: Allyn \& Bacon; 2003.

16. Ferrel $F$, Ferrel L, Cantillo $A$, Jaramillo J, Jiménez $S$. Variables académicas y sociodemográficas relacionadas con el Síndrome de Burnout, en estudiantes de Ingenierias y Ciencias de la Salud de una universidad estatal de Colombia . Psicogente. 2017; 20(38):336-352.

17. Martínez IM, Salanova Soria M. Niveles de Burnout y Engagement en estudiantes universitarios. Relación con el desempeño y desarrollo profesional. Rev Educ (Madrid). 2003; 330:361-384.

18. Salanova Soria M, Martínez Martínez IM, Bresó Esteve E, Llorens Gumbau S, Grau Gumbau R. Bienestar psicológico en estudiantes universitarios: facilitadores y obstaculizadores del desempeño académico. An Psicol. 2005; 21(1):170-180.

19. Santes M del C, Meléndez S, Martínez N, Ramos I, Preciado M de L, Pando M. La salud mental y predisposición a sindrome de burnout en estudiantes de enfermería. Rev Chil Salud Pública. 2009; 13(1):23-29.

20. Kinnunen JM, Lindfors P, Rimpelä A, Salmela-Aro K, Rathmann K, Perelman J, et al. Academic well-being and smoking among 14- to 17-year-old schoolchildren in six european cities. J Adolesc. 2016; 50:56-64.

21. Bolaños N, Rodriguez N. Prevalencia del Síndrome de Burnout académico en el estudiantado de Enfermería de la Universidad de Costa Rica. Enfermería Actual Costa Rica. 2016; 31:1-19. 